\title{
Colorectal Cancer Cells Activate Adjacent Fibroblasts Resulting in FGF1/FGFR3 Signaling and Increased Invasion
}

\author{
Maria L. Henriksson, ${ }^{*}$ Sofia Edin, ${ }^{*}$ \\ Anna M. Dahlin, ${ }^{*}$ Per-Arne Oldenborg, ${ }^{\dagger}$ \\ Åke Öberg, ${ }^{\ddagger}$ Bethany Van Guelpen, ${ }^{*}$ \\ Jörgen Rutegård, ${ }^{\ddagger}$ Roger Stenling, ${ }^{*}$ \\ and Richard Palmqvist* \\ From the Departments of Medical Biosciences, Pathology,* \\ Integrative Medical Biology, ${ }^{\dagger}$ Section for Histology and Cell \\ Biology, and Surgical and Perioperative Sciences, Surgery, ${ }^{\ddagger}$ Umeå \\ University, Umeå, Sweden
}

Cancer-associated fibroblasts expressing fibroblast activation protein (FAP) have been implicated in the invasive behavior of colorectal cancer. In this study, we use FAP expression as a marker of fibroblast activation and analyze the effect of activated fibroblasts on colorectal cancer migration and invasion in experimental cell studies. We also investigated the expression pattern of FAP in cancer-associated fibroblasts during transformation from benign to malignant colorectal tumors. In immunohistochemical analyses, FAP was expressed in fibroblasts in all colorectal cancer samples examined, whereas all normal colon, hyperplastic polyps, or adenoma samples were negative. In in vitro studies, conditioned medium from colon cancer cells, but not adenoma cells, activated fibroblasts by inducing FAP expression. These activated fibroblasts increased the migration and invasion of colon cancer cells in Boyden chamber experiments and in a three-dimensional cell culture model. We identify fibroblast growth factor 1 /fibroblast growth factor receptor 3 (FGF1/FGFR-3) signaling as mediators leading to the increased migration and invasion. Activated fibroblasts increase their expression of FGF1, and by adding a fibroblast growth factor receptor inhibitor, as well as an FGF1neutralizing antibody, we reduced the migration of colon cancer cells. Our findings provide evidence of a possible molecular mechanism involved in the cross talk between cancer cells and fibroblasts leading to cancer cell invasion. (Am J Pathol 2011, 178:1387-1394; DOI: 10.1016/j.ajpath.2010.12.008)
Colorectal cancer (CRC) is one of the leading causes of cancer deaths in western countries. Distant metastases represent the greatest threat to patient survival and about $40 \%$ of the CRC patients will die from a metastatic disease. Thus, although surgical resection is currently the basis for curative therapy, the need for nonsurgical treatment is being increasingly recognized. A detailed understanding of the biological processes regulating the development, progression, and metastasis of a malignant colorectal tumor may lead to improvements in nonsurgical anti-tumor therapy.

Awareness of the importance of the surrounding nonneoplastic stroma in tumor progression and invasion is increasing. The invasive behavior of tumors requires a number of cellular and molecular changes, not only in the epithelial cells, but also in the surrounding stroma. These changes include angiogenesis, lymphoid, and macrophage infiltration, release of cytokines/growth factors and proteolytic enzymes, and production of an altered extracellular matrix. ${ }^{1,2}$ There is also a complex interplay between the malignant cells and cancer-associated fibroblasts (CAFs), which are stromal cells that are abundantly present at the invasive front of primary colorectal tumors. A prominent component of CAFs is myofibroblasts. ${ }^{3,4}$ They have been suggested to produce pro-invasive signals that induce a more aggressive phenotype in the tumor ${ }^{5,6}$ CAFs are not transformed genetically, but differ from resting fibroblasts with regard to morphology, gene expression, and the production of important biological mediators, such as growth factors and proteases. ${ }^{7}$ They

Supported by the Swedish Cancer Society (grant CAN 2008/856); the Swedish Research Council (grant 521-20008-3994); the Cutting-Edge Research Grant from the County Council of Västerbotten, Sweden (grant VLL 159:41-2007); the Magn. Bergvalls Foundation; the Foundation Memory of Lars Hierta, Sweden; and the Cancer Research Foundation in Northern Sweden (grants LP-09-1798; AMP-08-554).

Accepted for publication December 7, 2010.

Supplemental material for this manuscript can be found at $h t t p: / / a j p$. amjpathol.org or at doi: 10.1016/j.ajpath.2010.12.008.

Address reprint requests to Maria Henriksson, Ph.D., Umeå University, Department of Medical Biosciences/Pathology, Building 6M, Floor 2, Umeå 901 85, Sweden. E-mail: maria.henriksson@medbio.umu.se. 
are often referred to as activated fibroblasts and have been seen to express the protease fibroblast activation protein (FAP)- $\alpha .{ }^{8} F A P$ is a membrane-bound glycoprotein belonging to the serine protease family that has been demonstrated to cleave gelatin and human collagen I, and therefore has been implicated in the remodeling of the extracellular matrix. ${ }^{9}$ It is normally expressed transiently in healing wounds, and abundantly by activated stromal fibroblasts in CRC and other human epithelial malignancies (breast, lung, and ovary carcinomas). ${ }^{10-12}$ Abundant FAP expression has been implicated as a negative prognostic factor in patients with metastatic $\mathrm{CRC},{ }^{13}$ but its biological role is still not clear. However, a clinical trial on CRC patients using talabostat, an inhibitor of FAP-enzymatic activity, showed a minimal clinical effect, and the authors suggest that the partial inhibition may be due to another contributing factor or factors. ${ }^{14}$

In the present study, we used FAP as a marker of activated fibroblasts to investigate their occurrence in various lesions of the adenoma-carcinoma sequence in human CRC. Further in vitro studies analyzed fibroblast activation in co-culture experiments. Additionally, a phospho-specific receptor, tyrosine kinase array, followed by blocking experiments explored the signals by which activated fibroblasts regulate colon cancer cell migration and invasion of extracellular matrix.

\section{Materials and Methods}

\section{Patients}

There were 156 archival colorectal tissue samples taken from a total of 125 patients that were analyzed. The tissue samples were collected between the years 1996 and 2005, and included 24 normal mucosa samples, 16 hyperplastic polyps, 30 tubular adenomas, 10 tubulovillous adenomas, 15 villous adenomas, and 61 adenocarcinomas. Twelve samples of normal mucosa and 6 tubular, 10 tubulovillous and 3 villous adenoma samples were located immediately adjacent to an adenocarcinoma within the same tissue block. The remaining 12 normal mucosa samples were collected distant to regions of adenocarcinoma but within the same surgical specimen, whereas the remaining adenoma and hyperplastic polyps samples came from patients with no adenocarcinoma. The local ethical committee approved the sample collection procedure and the use of samples for research purposes.

\section{Immunohistochemistry}

Specimens were fixed in $4 \%$ formaldehyde and embedded in paraffin, according to routine procedures at the Department of Clinical Pathology, Umeå University Hospital. One 4- $\mu \mathrm{m}$ section from each patient was cut, dried, de-waxed, and rehydrated. For FAP staining, slides were subjected to heat-mediated antigen retrieval using BORG solution (Histolab, Gothenburg, Sweden) in a Decloaker pressure cooker (Histolab). Anti-FAP monoclonal antibody (D8; Vitatex, Stony Brook, NY) was used at a concentration of 1:100, and Rat-on-Mouse HRP-Polymer kit (Biocare Medical, Concord, CA) was applied for detec- tion. For $\alpha$-smooth muscle actin (SMA) staining, slides were subjected to heat-mediated antigen retrieval using CC1 (Ventana Medical Systems Inc., Tuscan, AZ) in a Decloaker pressure cooker. Anti-SMA monoclonal antibody (clone 1A4; DAKO) was used at a concentration of 1:50 in a Ventana BenchMark ULTRA (Ventana Medical Systems Inc.). The slides were counterstained with hematoxylin.

For FAP evaluation, slides were reviewed under light microscopy. FAP immunostaining in fibroblasts was evaluated in $\mathrm{CRC}$ and in close vicinity to colonic epithelial cells in normal mucosa, hyperplastic polyps, and adenomas. Stromal staining was assessed as negative,,+++ , or +++ , according to the semiquantitative scale suggested by Henry et al. ${ }^{13}$ The,+++ and +++ categories were considered to be positive for FAP staining. Occasional epithelial staining was not analyzed. The specimens were evaluated twice by the same observer, and discordant cases were reviewed a third time followed by a conclusive judgment. The reviewer was blinded to the identity of the specimens between the first and second evaluation.

\section{Cell Lines and Cell Culture}

The colon cancer cell line (HCT-116) and fibroblasts from normal colon (CCD-18Co) (both from American Type Culture Collection, Manassas, VA) were grown in DMEM supplemented with $10 \%$ heat-inactivated fetal bovine serum (Gibco, Invitrogen, Paisley, UK) at $37^{\circ} \mathrm{C}$ and $5 \%$ carbon dioxide. The colon adenoma cell line (LT97) (a generous gift from Dr. Brigitte Marian, Vienna, Austria) were cultured as previously described. ${ }^{15}$

For conditioned medium experiments, attached cells were rinsed twice in PBS and fresh medium was added. In the case of LT97, fibroblasts were pre-cultured in LT97 medium to eliminate effects of medium content. Flasks were returned to $37^{\circ} \mathrm{C}$ with $5 \%$ carbon dioxide. After 48 hours, the conditioned medium was collected and used either in migration assays or in the organotypic cell culture invasion assay. If the conditioned medium was not immediately used, it was frozen at $-80^{\circ} \mathrm{C}$. In each experiment, conditioned medium from the same batch was used to assure that different results did not depend on differently activated cells.

To induce FAP expression, and thereby activate CCD18Co fibroblasts, we stimulated with conditioned medium from colon cancer cells or $10 \mathrm{ng} / \mathrm{ml}$ tumor growth factor (TGF) $\beta-1$ (R\&D Systems, Minneapolis, MN) for 3 days.

\section{Immunofluorescence}

CCD-18Co fibroblasts cultured on glass coverslips were washed briefly with PBS, fixed (3\% formaldehyde; $2 \%$ sucrose; PBS) for 10 minutes at room temperature, and washed in PBS/0.1 mol/L glycine. For permeabilization and block, the cells were treated with PBS containing $10 \%$ fetal bovine serum, $0.4 \%$ Triton $X-100$ for 20 minutes, followed by wash in wash buffer (PBS; 0.2\% Triton $X-100 ; 0.2 \%$ bovine serum albumin). The cells were then incubated with FAP primary antibody (diluted 1:100 in PBS; $0.1 \%$ Triton $\mathrm{X}$-100) for 1 hour at room temperature, 
after which the coverslips were washed in wash buffer. The cells were further incubated with a secondary antibody (diluted 1:400 in PBS; 0.1\% Triton X-100) for 1 hour. An additional wash was performed, after which the coverslips were mounted in Vectashield mounting medium (Vector Laboratories, Burlingame, CA). Cells were viewed using a Nikon D-Eclipse C1 confocal microscope (Nikon, Langen, Germany), using oil immersion and $\times 40$ objective.

\section{Morphology}

The cells were cultured for 3 hours in normal cell culture medium or conditioned medium from activated (FAP-expressing) or resting (FAP-negative) CCD-18Co fibroblasts with or without the fibroblast growth factor receptor (FGFR)-inhibitor PD173074 (SIGMA). Live photography was taken with a DeltaPix Invenio 3S (DeltaPix Aps, Maalov, Denmark) mounted on a Leitz Diavert microscope (Esselte, Stamford, CT).

\section{Migration}

Boyden chamber experiments were performed to analyze how activated fibroblasts (FAP-expressing) influence the migration of cancer cells. There were 100,000 HCT116 cells or LT97 cells that were placed in a 24-well cell culture insert (8- $\mu \mathrm{m}$ pore size; BD Biosciences, San Jose, CA) in 500- $\mu$ l culture medium and were allowed to adhere for 3 hours. Next, culture inserts were placed in conditioned medium (from $+/-$ TGF $\beta$ - 1 stimulated CCD$18 \mathrm{Co}$ cells), or medium containing either $10 \%$ fetal bovine serum or no serum (positive and negative control) and incubated for 20 hours. After being washed in PBS, the inserts were placed in ice-cold methanol for 1 minute and washed again in PBS. Cells adhering to the inside of the insert were scraped off with a cotton swab, and the cells on the outside were stained with $0.5 \%$ Coomassie blue. After washes in PBS, the filter was cut out and the cells were counted in three randomly selected fields. The migration index sets the positive control as 1 . The experiment was repeated at least 3 times.

\section{Organotypic Cell Culture Invasion Assay}

To study the capacity of the epithelial cells to degrade and invade extracellular matrix, we developed a threedimensional invasion assay. One volume containing CCD-18Co fibroblasts $(1,000,000 / \mathrm{ml})$ was suspended in matrix [seven volumes collagen I/Matrigel mixture (BD Biosciences, San Jose, CA), one volume culture medium, one volume fetal bovine serum], and overlaid with culture medium containing HCT116 or LT97 cells. In experiments in which there were no fibroblasts used, one volume of culture medium or conditioned medium was used instead. After 7 to 14 days, organotypic cell culture invasion assays were fixed in $4 \%$ formaldehyde, paraffin-embedded, and sectioned for staining with H\&E, or pre-treated with protease, followed by antibodies directed at cytokeratin Cam5.2 (BD Biosciences) using a semi-automatic staining machine (Ventana Medical Systems) to visualize epithelial cells.

\section{Proteome Profiler Array}

The Proteome profiler array (R\&D Systems, Minneapolis, $\mathrm{MN}$ ) were performed according to the manufacturers' instructions. Briefly, HCT116 tumor cells were starved overnight, followed by stimulation for 15 minutes with conditioned medium from fibroblasts, pre-activated with TGF $\beta-1$, or not. The cells were lysed with lysis buffer ( $1 \%$ $\mathrm{NP}-40,20 \mathrm{mmol} / \mathrm{L}$ Tris- $\mathrm{HCl} \mathrm{pH}$ 8.0, $137 \mathrm{mmol} / \mathrm{L} \mathrm{NaCl}$, $10 \%$ glycerol, $2 \mathrm{mmol} / \mathrm{L}$ EDTA, $1 \mathrm{mmol} / \mathrm{L}$ sodium orthovanadate, $10 \mu \mathrm{g} / \mathrm{ml}$ aprotinin, $10 \mu \mathrm{g} / \mathrm{ml}$ leupeptin). Array filters were incubated with respective lysate overnight, and after washes were followed by detection antibody included in the kit. Finally, after repeated washing, the array was exposed to chemiluminiscent reagents (Pierce, VWR International, Stockholm, Sweden).

\section{TGF $\beta-1$ ELISA}

The level of TGF $\beta$ - 1 in conditioned medium was measured by enzyme-linked immunosorbent assay [(ELISA) Quantikine kit, R\&D Systems] according to the manufacturer's instructions.

\section{Western Blot}

To analyze FAP expression in TGF $\beta$ - 1 activated CCD$18 \mathrm{Co}$ fibroblasts, cells were lysed in lysis buffer (100 $\mathrm{mmol} / \mathrm{L} \mathrm{NaCl}, 50 \mathrm{mmol} / \mathrm{L}$ Tris $\mathrm{pH}$ 7.5, 1\% Triton X-100, 1 $\mathrm{mmol} / \mathrm{L}$ EDTA $\mathrm{pH}$ 8, protease inhibitors). Proteins were separated using SDS-PAGE and immunoblotting was performed according to the manufacturer's instructions, using secondary antibodies conjugated to horseradish peroxidase (GE Healthcare, Uppsala, Sweden). Blots were developed with SuperSignal West Pico Chemiluminescent Substrate (Pierce). FAP antibody was used at 1:500, anti-SMA (DAKO) was used at 1:200.

\section{Statistics}

To test the differences between experimental groups the nonparametric Mann-Whitney U-test was performed. A significance level of 0.05 was used.

\section{Results}

\section{Fibroblasts in Close Vicinity to Colorectal Cancer Selectively Express FAP}

Immunohistochemistry using a monoclonal FAP antibody was performed on 156 sections obtained from formalinfixed, paraffin-embedded samples of normal human coIon mucosa, tubular, tubulovillous, and villous adenomas, hyperplastic polyps, and colorectal adenocarcinomas. FAP immunoreactivity was generally seen in CAFs only. No fibroblasts adjacent to normal colonic mucosa, hyperplastic polyps, or adenomas demonstrated any positivity 

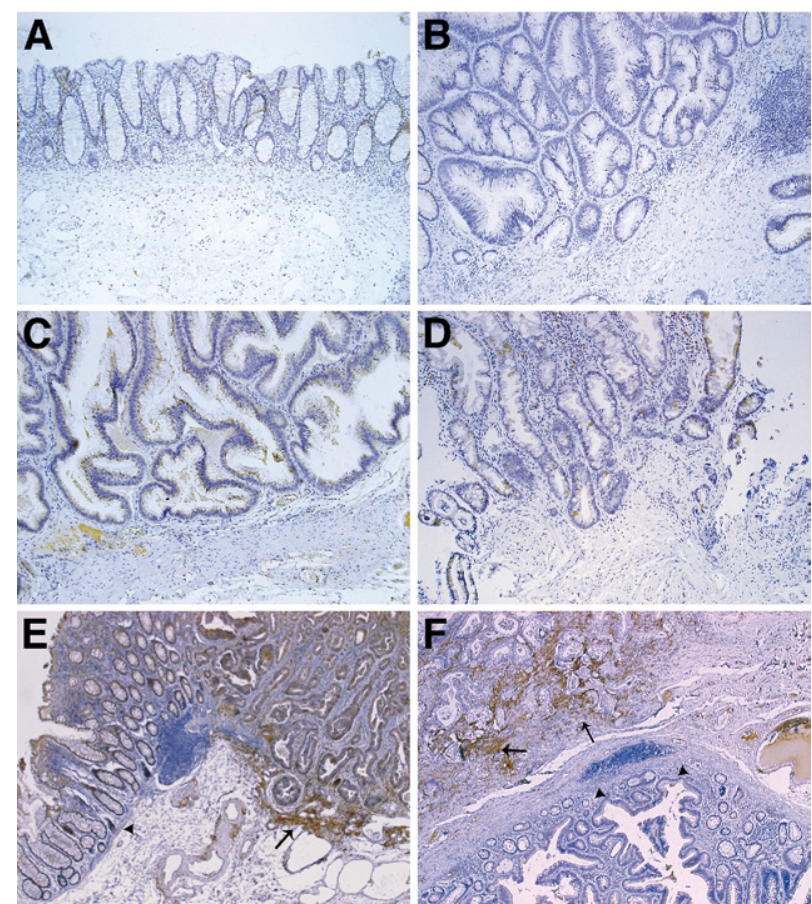

Figure 1. Fibroblast activation protein (FAP)-expressing fibroblasts are found in close proximity to colon carcinoma. Representative examples of FAP expression by immunohistochemistry. Normal colonic tissue (A); tubular adenoma (B); villous adenoma (C); hyperplastic polyp (D); the border between normal colon mucosa and colon adenocarcinoma $(\mathbf{E})$; the border between adenoma and colon adenocarcinoma (F). Arrows are pointing at FAP-positive fibroblasts (E and $\mathbf{F}$ ), and arrowheads are pointing at normal colon mucosa (E) and adenoma (F). FAP antibody was used at a concentration of $1: 100$.

at all (Figure 1, A-D). Importantly, in all cases in which normal colonic mucosa or adenoma tissue was located immediately adjacent to adenocarcinoma tissue, a clearcut border was seen between the FAP-negative fibroblasts associated with benign tissue and the FAP-positive fibroblasts associated with malignant tissue (Figure 1, E and F). Detailed results are presented in Table 1. A commonly used marker for activated fibroblasts is $\alpha$-SMA. Figure 2 shows that (in contrast to FAP-staining) the SMA antibody not only stains CAFs, but also fibroblasts adjacent to adenomas and smooth muscle cells in muscularis

Table 1. FAP Expression in Fibroblasts in Different Colorectal Lesions

\begin{tabular}{lcccc}
\hline & FAP & \multicolumn{2}{c}{ FAP positive } \\
\cline { 3 - 5 } & negative & + & ++ & +++ \\
& 12 & 0 & 0 & 0 \\
Normal mucosa & 12 & 0 & 0 & 0 \\
Normal mucosa adjacent & 16 & 0 & 0 & 0 \\
to CRC & 30 & 0 & 0 & 0 \\
Hyperplastic polyps & 15 & 0 & 0 & 0 \\
Tubular adenomas & 10 & 0 & 0 & 0 \\
Villous adenomas & 19 & 0 & 0 & 0 \\
Tubulovillous adenomas & & & & \\
Adenoma adjacent to & 0 & 15 & 19 & 27 \\
$\quad$ CRC & & & & \\
Colorectal cancer & & &
\end{tabular}

+ , weak; ++ , moderate; +++ , strong FAP staining; CRC, colorectal cancer; FAP, fibroblast activation protein

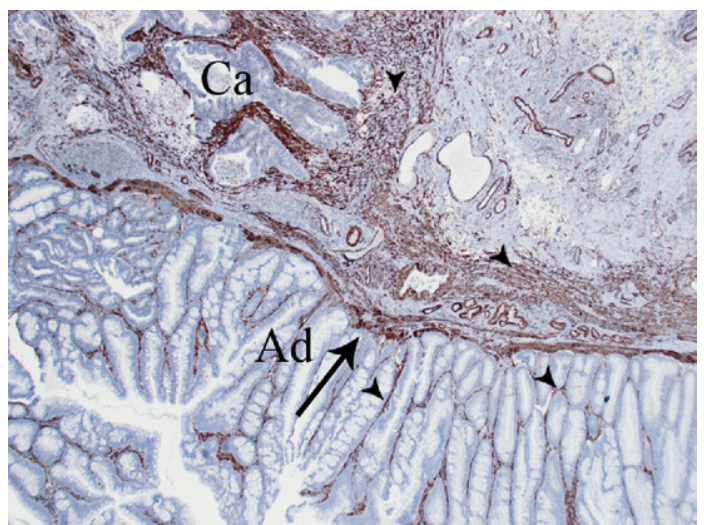

Figure 2. $\alpha$-Smooth muscle actin (SMA) expression in colorectal cancer and adenoma. Representative example of $\alpha$-SMA expression by immunohistochemistry. Arrow is pointing at smooth muscle cells in mucularis mucosae, and arrowheads are pointing at fibroblasts in close vicinity to both adenoma and invasive cancer. Ad, adenoma; $\mathrm{Ca}$, invasive cancer.

mucosae. Hereafter, we use FAP as a marker for activated fibroblasts.

\section{FAP Expression in Fibroblasts Is Induced by Colon Cancer Cells in Vitro}

We cultured normal colonic CCD-18Co fibroblasts with conditioned medium from the HCT116 cancer cell line or the nonmalignant LT97 adenoma cell line for 3 days. Using a monoclonal antibody against FAP in fluorescent staining, we found that conditioned medium from HCT116 cells activated the fibroblasts, as revealed by an increased FAP expression (Figure 3B), whereas conditioned medium from LT97 cells did not (Figure 3C). Measuring TGF $\beta-1$ content in the conditioned medium showed that HCT116 cells produced more than 15 times
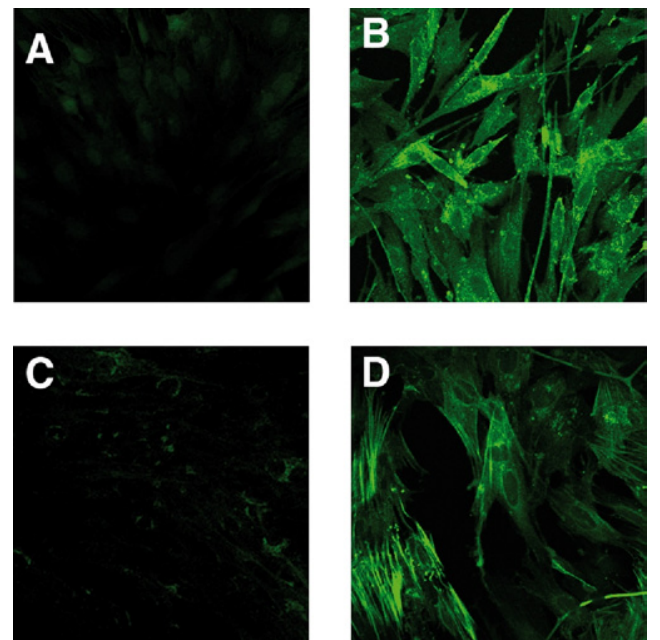

Figure 3. Colon cancer cells induce fibroblast activation protein (FAP) expression in fibroblasts. Immunofluorescent staining of FAP in CCD-18Co fibroblasts. Fibroblasts were incubated for 3 days in: normal cell culture medium (A), conditioned medium from HCT116 colon cancer cells (B), conditioned medium from LT97 adenoma cells $(\mathbf{C})$, and normal cell culture medium supplemented with TGF $\beta-1$ (10 ng/ml, D) ( $\times 40$ objective magnification was used). The green color corresponds to FAP expression. 
more TGF $\beta$ - 1 than the LT97 cells (see Supplemental Figure $\mathrm{S} 1$ at $h$ ttp://ajp.amjpathol.org). Indeed, in vitro stimulation with TGF $\beta$-1 also activated CCD-18Co fibroblasts, seen with both fluorescent staining (Figure 3D) and Western blot analysis (see Supplemental Figure S2 at $h t t p: / /$ ajp.amjpathol.org) using an FAP antibody, indicating that TGF $\beta$-1 stimulated fibroblasts can be used as a suitable model for further studies of activated, FAP-expressing, fibroblasts in vitro. We used SMA as a control to show that the FAP-expressing fibroblasts are indeed activated (see Supplemental Figure S2 at http://ajp.amjpathol.org).

\section{Activated Fibroblasts Promote Morphological Changes and Migration of Colon Cancer Cells}

When culturing HCT116 colon cancer cells in conditioned medium from activated CCD-18Co fibroblasts, a clear change in morphology was already seen after 3 hours (Figure 4A). HCT116 cells grown in conditioned medium from activated fibroblasts showed a more elongated morphology (Figure 4A, top row, right panel) compared to

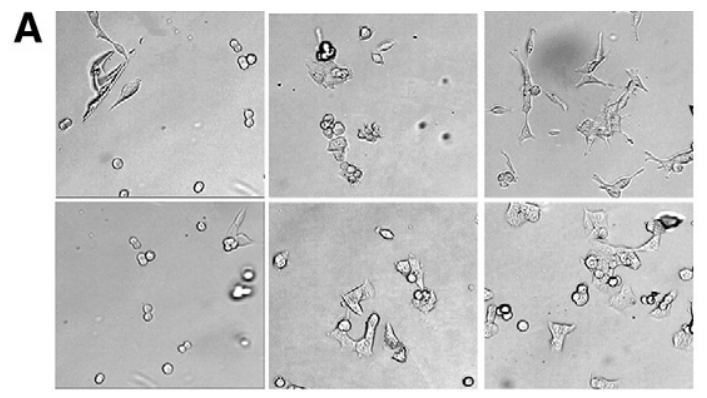

B

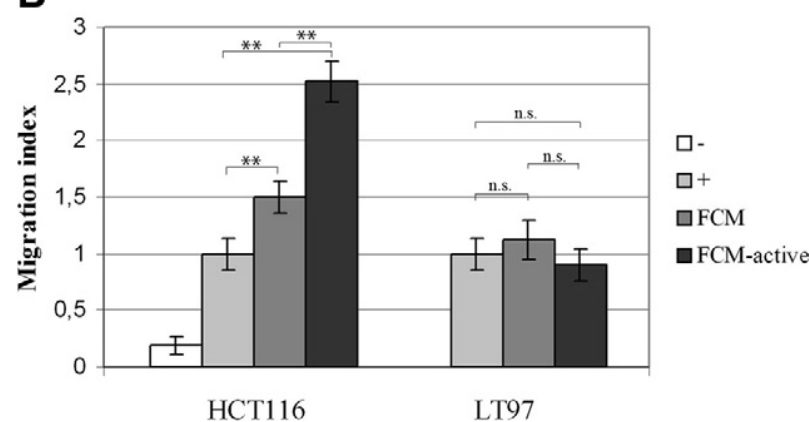

Figure 4. Conditioned medium from activated fibroblasts induces morphological changes and increased migration of colon cancer cells. A: HCT116 colon cancer cells were cultured for 3 hours with (top row, left panel) normal culture medium; (top row, middle panel) conditioned medium from resting CCD-18Co fibroblasts; (top row, right panel) conditioned medium from tumor growth factor (TGF) $\beta-1$ (10 ng/ml) activated CCD-18Co fibroblasts; (bottom row, left panel) normal culture medium containing the fibroblast growth factor receptor (FGFR) inhibitor PD173074; (bottom row, middle panel) conditioned medium from resting CCD-18Co fibroblasts containing the FGFR-inhibitor PD173074; and (bottom row, right panel) conditioned medium from TGF $\beta-1$ ( $10 \mathrm{ng} / \mathrm{ml})$ activated CCD-18Co fibroblasts $(\times 4$ objective magnification was used). B: Boyden chamber experiment showing the migration of HCT116 or LT97 adenoma cells. (-), starvation medium; $(+)$, normal cell culture medium; fibroblast conditioned medium (FCM), conditioned medium from resting CCD-18Co fibroblasts; FCM-active, conditioned medium from CCD-18Co fibroblasts activated by TGF $\beta-1(10 \mathrm{ng} / \mathrm{ml})$. Migration toward normal cell culture medium was set as 1 in the migration index. ${ }^{* *} P<0.01$; n.s., not statistically significant. cells growing in normal cell culture medium (Figure 4A, top row, left panel) or conditioned medium from resting fibroblasts (Figure 4, top row, middle panel). To study if these morphological changes were indicating increased cell motility, a Boyden chamber migration assay was performed, in which HCT116 cells were allowed to migrate toward either starvation medium, culture medium containing fetal bovine serum, conditioned medium from resting fibroblasts, or conditioned medium from activated fibroblasts. As shown in Figure 4B, conditioned medium from fibroblasts, especially from activated fibroblasts, significantly increased the migration of the cancer cells compared to ordinary cell culture medium by approximately 2.5 times. When the LT97 adenoma cell line was used in the same assay, no increased migration was seen with conditioned media from fibroblasts (Figure 4B).

\section{Activated Fibroblasts Promote Invasion of Colon Cancer Cells in a Three-Dimensional Organotypic Cell Culture Model}

We wanted to determine whether CCD-18Co fibroblasts could induce HCT116 colon cancer cell invasion of extracellular matrix. We performed a three-dimensional organotypic cell culture model. Resting or activated (FAPexpressing) fibroblasts were embedded in a mixture of Matrigel (BD Biosciences) and collagen I, on which HCT116 cells or LT97 adenoma cells were seeded. After 1 week, the cultures were harvested, fixated in formalin, and paraffin embedded. Neither HCT116 nor LT97 cells invaded the Matrigel/collagen I mixture in the absence of fibroblasts (Figure 5, A and B). A small increase in migration was seen for HCT116 cells when resting fibroblasts were embedded within the matrix (Figure 5C). When activated CCD-18Co fibroblasts were used instead, the HCT116 cells demonstrated a strong invasive phenotype (Figure 5D). We also immunohistologically stained the sections with an anti-cytokeratin CAM5.2 antibody to clearly distinguish the invading cancer cells from surrounding fibroblasts (Figure 5, E and F). In contrast, the LT97 cells did not invade the matrix containing either resting or activated fibroblasts, (Figure 5, G and $\mathrm{H}$ ), not even after an extended co-cultivation of 2 weeks (data not shown). FAP, the marker for activated fibroblasts previously used, is localized to the fibroblast membrane. To determine whether the induced invasion by HCT116 cells was dependent on FAP protease activity, we embedded conditioned medium from resting or activated fibroblasts in the Matrigel/collagen I mixture, without any fibroblasts present (Figure 5, I and J). After 1 week of incubation, the HCT116 cells had begun to invade the matrix containing conditioned medium from activated fibroblasts (Figure 5J), indicating that the increased invasive capacity was at least partly independent of FAP protease activity. HCT116 cells cultured with conditioned medium from resting fibroblasts did not invade the matrix (Figure 5I). 

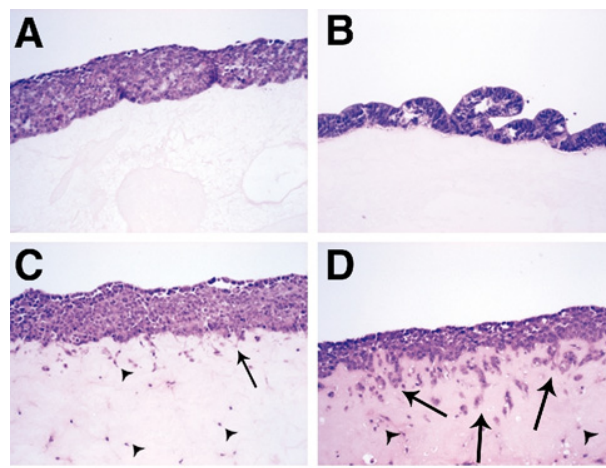

$\mathbf{E}$

D
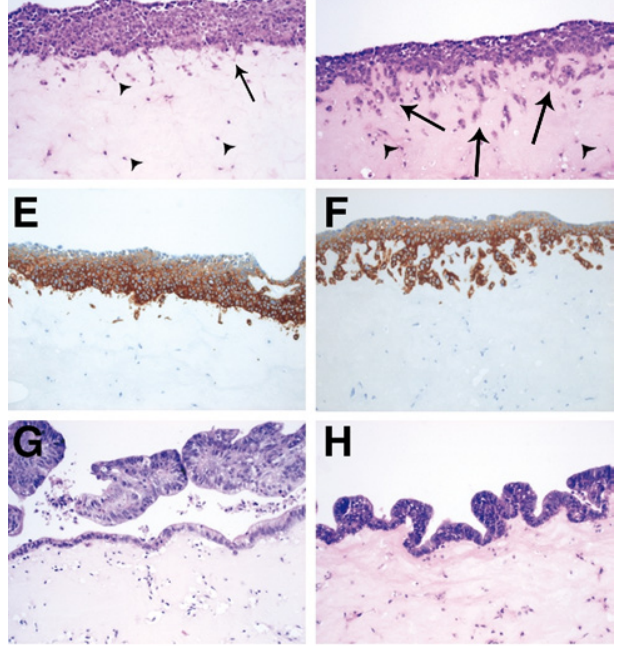

$\mathrm{H}$

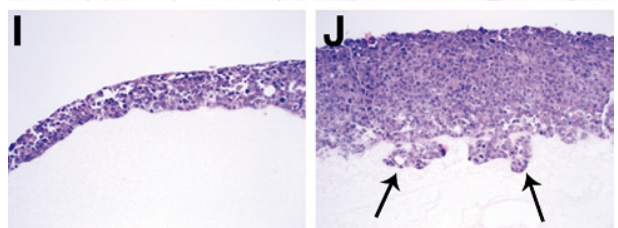

Figure 5. Activated fibroblasts induce colon cancer cell invasion of extracellular matrix. An organotypic cell culture model showing the invasion of colon cancer cells. HCT116 colon cancer cells cultured without CCD-18Co fibroblasts (A); LT97 adenoma cells cultured without CCD-18Co fibroblasts (B) : HCT116 cells co-cultured with resting CCD-18Co fibroblasts (C); HCT116 cells co-cultured with CCD-18Co fibroblasts activated by tumor growth factor (TGF) $\beta-1(10 \mathrm{ng} / \mathrm{ml})$ (D); HCT116 cells co-cultured with resting CCD-18Co fibroblasts, epithelial cells are visualized by immunohistological staining with anti-cytokeratin Cam5.2 (E); HCT116 cells co-cultured with CCD-18Co fibroblasts activated by TGF $\beta-1(10 \mathrm{ng} / \mathrm{ml})$, and epithelial cells are visualized by immunohistological staining with anti-cytokeratin Cam5.2 (F); LT97 cells cultured together with resting CCD-18Co fibroblast (G); LT97 cells cocultured with CCD-18Co fibroblasts activated by TGF $\beta-1(10 \mathrm{ng} / \mathrm{ml})(\mathbf{H})$ HCT116 cells cultured with conditioned medium from resting CCD-18Co fibroblasts (I); and HCT116 cells cultured with conditioned medium from CCD-18Co fibroblasts activated by TGF $\beta-1(10 \mathrm{ng} / \mathrm{ml})(\mathbf{J})$. Arrows are showing invading tumor cells and arrowheads are showing fibroblasts.

\section{Morphological and Migratory Changes of Colon Cancer Cells Are Dependent on FGFR3 and FGF1 from Activated Fibroblasts}

To identify the factor or factors in conditioned medium from fibroblasts that induce the increased migration and invasion, we performed a Proteome Profiler array (R\&D Systems). Fibroblast growth factor receptor 3 (FGFR3) was identified as one of the receptor tyrosine kinases activated by conditioned medium from activated (FAP expressing) fibroblasts (Figure 6A). A detailed description of all receptors phosphorylated is shown (see Supplemental Figure S3 at http://ajp.amjpathol.org). When culturing HCT116 colon cancer cells with conditioned medium from activated CCD-18Co fibroblasts, including an FGFR-inhibitor (PD173074), the morphological changes of the cancer cells previously shown in Figure $4 \mathrm{~A}$ was abolished (compare top row of right panel, and bottom row of right panel), and the increased migration with conditioned medium from activated CCD-18Co shown in Figure 4B was also attenuated (Figure 6B). One of the ligands for FGFR3 is fibroblast growth factor 1 (FGF1). Real-time semi-quantitative PCR analysis of FGF1 expression in CCD-18Co fibroblasts revealed that FGF1 expression was increased by approximately 1.7fold in activated compared to resting fibroblasts (data not shown). Therefore, we continued by neutralizing FGF1 in the conditioned medium from activated fibroblasts to analyze whether the increased migration of the cancer cells was dependent on FGF1. When adding a FGF1-neutralizing antibody to the conditioned medium from activated fibroblasts, we indeed could see a reduced migration (Figure 6B).

\section{Discussion}

Several studies indicate that tumor progression and invasion is determined not only by the malignant cancer cells, but also by the surrounding non-neoplastic stromal cells, including fibroblasts. Activated fibroblasts (expressing FAP) have been described to be present in CAFs in several epithelial malignancies, including $\mathrm{CRC}^{10,13,16}$.

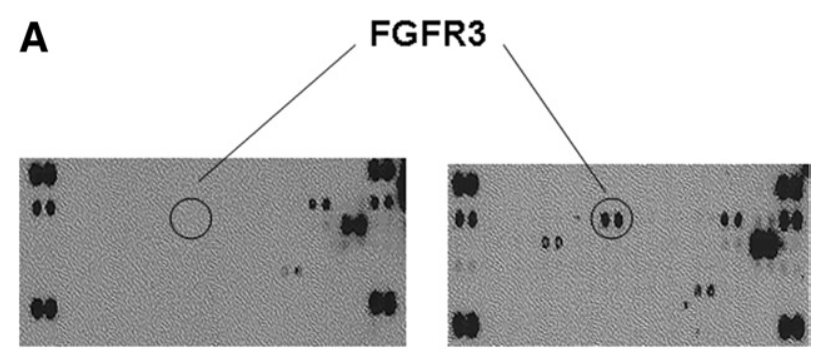

B

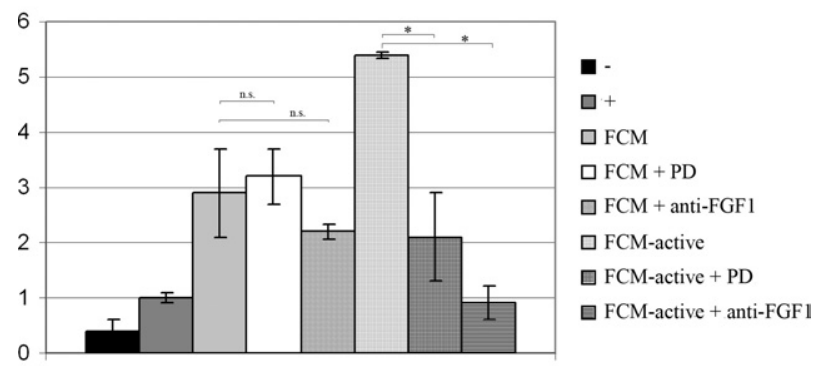

Figure 6. Fibroblast growth factor receptor 3 (FGFR) and fibroblast growth factor 1 (FGF1) partly regulate migration in colon cancer cells. A: Proteome profiler array (R\&D Systems, Minneapolis, MN) showing receptor tyrosine kinase receptors phosphorylated after stimulation with conditioned medium from resting CCD-18Co fibroblasts (left) and stimulation with conditioned medium from TGF $\beta-1$ activated CCD-18Co fibroblasts (right). Phosphorylation of FGFR3 is highlighted with circles (see Supplemental Figure S3 at bttp://ajp.amjpathol.org), which specifies all of the phosphorylated receptor tyrosine kinases. B: Boyden chamber experiment showing the migration of HCT116 cells. (-), starvation medium; (+), normal cell culture medium; fibroblast conditioned medium (FCM), conditioned medium from resting CCD-18Co fibroblasts; FCM-active, conditioned medium from CCD-18Co fibroblasts activated with tumor growth factor (TGF) $\beta-1(10 \mathrm{ng} / \mathrm{ml}) ; \mathrm{PD}$, FGFR-inhibitor PD173074; anti-FGF1, FGF1-neutralizing antibody. Migration toward ordinary culture medium was set as 1 in the migration index. ${ }^{*} P<$ 0.05 ; n.s., not statistical significant. 
We have shown in the present study, that FAP can be used as a marker for invasive CRC. However, our data indicates that the ability of FAP-positive activated fibroblasts to promote migration of malignant epithelial cells in vitro may to a lesser extent be associated with FAP per se, but instead be the result of FGF1 released by FAPpositive fibroblasts, acting on FGFR on the epithelial cells.

First we show, in the largest study to date, that normal colonic mucosa, tubular, tubulovillous and villous adenomas, and hyperplastic polyps lack FAP-positive fibroblasts. Fibroblasts bordering the colorectal cancers, on the other hand, consistently demonstrate positivity for FAP. Our findings are in agreement with a previous, smaller study. ${ }^{10}$ At present, no established objective marker for early invasion exists. Such a diagnostic tool is eagerly being awaited because distinguishing early CRC invasion can be diagnostically difficult, both in the small biopsy specimen and adenoma tissue. Expression of SMA is commonly used as a marker for activated fibroblasts. A drawback with this marker is that this antibody does not only stain CAFs but also adenoma-associated fibroblasts and smooth muscle cells, making it difficult to distinguish an invasive cancer from a noninvasive one (Figure 2). Because FAP expression is clearly upregulated during the transformation of benign colorectal tissue into cancer, we suggest that FAP may be a useful diagnostic marker for early tumor invasion in CRC.

The finding of a clear border of activated fibroblasts between normal colonic mucosa and adenocarcinoma may infer that fibroblast activation is induced by the cancer cells themselves. Here we show that conditioned medium from cancer cells, but not from adenoma cells, can indeed induce activation of fibroblasts in vitro.

We further show that these activated fibroblasts increase the migratory capacity of colon cancer cells in vitro by producing FGF1. To our knowledge, this is the first study showing that FAP-positive fibroblasts can produce soluble factor or factors that affect the behavior of the tumor cells. FGF1 is a pleiotropic growth factor known to regulate proliferation, migration, and differentiation of target cells, and it has been shown to promote intestinal epithelial cell migration. ${ }^{17} \mathrm{~A}$ recent article has additionally shown that overexpression of FGFR correlates with liver metastasis in CRC, ${ }^{18}$ and the splice form IIIc of FGFR3 has been suggested to have oncogenic effect on CRC cells. ${ }^{19}$ Because both a block of FGFR and use of a neutralizing FGF1 antibody attenuated cancer cell migration in our assay, we speculate that FGF signaling might be one pathway responsible for the low clinical efficiency seen when using the FAP-inhibitor talabostat on metastatic CRC patients. ${ }^{14}$ This is the first study showing that activated fibroblasts produce FGF1, and thereby increase colon cancer cell migration, and the data suggests that future analysis should focus on the interplay between cancer and stroma cells.

Activated fibroblasts induce a more fibrotic stroma, as seen in several cancers, including CRC. Interestingly, during the development of lung fibrosis, FGF1 has recently been shown to inhibit myofibroblast differentiation. ${ }^{20}$ This could suggest an autocrine FGF signaling in fibroblasts that might be important also during cancer progression. However, in this study we have only focused on FGF1 signaling effects in cancer cells, and further studies are needed to clarify this field.

In our organotypic cell culture model, we found that activated fibroblasts and (perhaps more importantly) conditioned medium from activated fibroblasts also increase the invasion of colon cancer cells through the extracellular matrix. High levels of FAP immunoreactivity have been positively correlated with the invasive behavior of several malignant tumors, and abundant expression has been associated with lymph node metastasis in CRC. ${ }^{16}$ Most previous studies have focused on FAP protease activity, which degrades extracellular matrix molecules, thereby creating a milieu more conductive to invasion as the cause of increased invasion. However, FAP is a membrane-bound protease and should not be present in the conditioned medium from activated fibroblasts. Therefore, our migration assay and organotypic cell culture experiments both provide strong evidence that the physical presence of FAP, and thus its protease activity, is not the only mechanism by which activated fibroblasts promote migration and invasion. The interplay between cancer cells and fibroblasts results in fibroblast-secreted factors, such as FGF1, leading to an overall invasive behavior of the cancer cells.

In conclusion, we show that FAP-expressing, activated fibroblasts are present in CRC, but not in premalignant colonic lesions. Therefore, our data suggest that FAP may be a potentially valuable clinical diagnostic marker for early tumor invasion in colorectal tissue. In vitro, for the first time, we show that tumor cells have the capacity to induce FAP expression in fibroblasts in CRC. We also demonstrate that activated fibroblasts promote migration of malignant epithelial cells via FGF1/FGFR; further analysis of activated fibroblasts is needed to pinpoint the role during CRC progression.

\section{Acknowledgments}

The authors thank Professor Brigitte Marian (Institute of Cancer Research, University of Vienna, Austria) for the generous gift of LT97 adenoma cells and Mrs. Kerstin Näslund (Department of Medical Biosciences, Pathology, Umeå University, Sweden) for her skillful technical assistance.

\section{References}

1. Seljelid R, Jozefowski S, Sveinbjornsson B: Tumor stroma. Anticancer Res 1999, 19:4809-4822

2. Almholt K, Johnsen M: Stromal cell involvement in cancer. Recent Results Cancer Res 2003, 162:31-42

3. Bhowmick NA, Neilson EG, Moses HL: Stromal fibroblasts in cancer initiation and progression. Nature 2004, 432:332-337

4. Weinberg R: The biology of cancer. New York, Garland Science, 2007: pp 537-551

5. Liang P, Hong JW, Ubukata H, Liu G, Katano M, Motohashi G, Kasuga T, Watanabe Y, Nakada I, Tabuchi T: Myofibroblasts correlate with lymphatic microvessel density and lymph node metastasis in earlystage invasive colorectal carcinoma. Anticancer Res 2005, 25:27052712 
6. Nakayama H, Enzan H, Miyazaki E, Naruse K, Kiyoku H, Hiroi M: The role of myofibroblasts at the tumor border of invasive colorectal adenocarcinomas. Jpn J Clin Oncol 1998, 28:615-620

7. Kalluri R, Zeisberg M: Fibroblasts in cancer. Nat Rev Cancer 2006 6:392-401

8. Scanlan MJ, Raj BK, Calvo B, Garin-Chesa P, Sanz-Moncasi MP, Healey $\mathrm{JH}$, Old LJ, Rettig WJ: Molecular cloning of fibroblast activation protein alpha, a member of the serine protease family selectively expressed in stromal fibroblasts of epithelial cancers. Proc Natl Acad Sci USA 1994, 91:5657-5661

9. Aggarwal S, Brennen WN, Kole TP, Schneider E, Topaloglu O, Yates M, Cotter RJ, Denmeade SR: Fibroblast activation protein peptide substrates identified from human collagen I derived gelatin cleavage sites. Biochemistry 2008, 47:1076-1086

10. Garin-Chesa P, Old LJ, Rettig WJ: Cell surface glycoprotein of reactive stromal fibroblasts as a potential antibody target in human epithelial cancers. Proc Natl Acad Sci USA 1990, 87:7235-7239

11. Rettig WJ, Su SL, Fortunato SR, Scanlan MJ, Raj BK, Garin-Chesa P Healey JH, Old LJ: Fibroblast activation protein: purification, epitope mapping and induction by growth factors. Int J Cancer 1994, 58:385392

12. Rettig WJ, Garin-Chesa P, Beresford HR, Oettgen HF, Melamed MR, Old LJ: Cell-surface glycoproteins of human sarcomas: differential expression in normal and malignant tissues and cultured cells. Proc Natl Acad Sci USA 1988, 85:3110-3114

13. Henry LR, Lee HO, Lee JS, Klein-Szanto A, Watts P, Ross EA, Chen WT, Cheng JD: Clinical implications of fibroblast activation protein in patients with colon cancer. Clin Cancer Res 2007, 13:1736-1741
14. Narra K, Mullins SR, Lee HO, Strzemkowski-Brun B, Magalong K, Christiansen VJ, McKee PA, Egleston B, Cohen SJ, Weiner LM, Meropol NJ, Cheng JD: Phase II trial of single agent Val-boroPro (Talabostat) inhibiting fibroblast activation protein in patients with metastatic colorectal cancer. Cancer Biol Ther 2007, 6:1691-1699

15. Richter M, Jurek D, Wrba F, Kaserer K, Wurzer G, Karner-Hanusch J, Marian B: Cells obtained from colorectal microadenomas mirror early premalignant growth patterns in vitro. Eur J Cancer 2002, 38:19371945

16. Iwasa S, Jin X, Okada K, Mitsumata M, Ooi A: Increased expression of seprase, a membrane-type serine protease, is associated with lymph node metastasis in human colorectal cancer. Cancer Lett 2003, 199:91-98

17. Dignass AU, Tsunekawa S, Podolsky DK: Fibroblast growth factors modulate intestinal epithelial cell growth and migration. Gastroenterology 1994, 106:1254-1262

18. Takayama T, Miyanishi K, Hayashi T, Sato Y, Niitsu Y: Colorectal cancer: genetics of development and metastasis. J Gastroenterol 2006, 41:185-192

19. Sonvilla G, Allerstorfer S, Heinzle C, Stattner S, Karner J, Klimpfinger M, Wrba F, Fischer H, Gauglhofer C, Spiegl-Kreinecker S, GraslKraupp B, Holzmann K, Grusch M, Berger W, Marian B: Fibroblast growth factor receptor 3-IIlc mediates colorectal cancer growth and migration. Br J Cancer 102:1145-1156

20. Ramos C, Montano M, Becerril C, Cisneros-Lira J, Barrera L, Ruiz V, Pardo A, Selman M: Acidic fibroblast growth factor decreases alphasmooth muscle actin expression and induces apoptosis in human normal lung fibroblasts. Am J Physiol Lung Cell Mol Physiol 2006, 291:L871-879 